\title{
Electrical Conductivity and Moisture Uptake Studies of Low Density Polyethylene Octylnanosilica Composite
}

\author{
S. Virtanen, A.S. Vaughan \\ ECS; University of Southampton \\ Southampton, United Kingdom \\ S.Yang, F. Saiz, N. Quirke \\ Department of Chemistry, Imperial College, \\ London, United Kingdom
}

\begin{abstract}
In this paper, we report on the time dependent DCconductivity response at constant electric field of unfilled low density polyethylene (LDPE) and its composites containing untreated and octylsilane treated silica in ambient and dry atmospheres. The conductivity of both composites decrease with time under dry conditions and increase with time in ambient atmosphere. The dielectric response of dry and wet silica polyethylene composites is studied by dielectric spectroscopy.
\end{abstract}

\section{Introduction}

DC-conductivity is considered to be one of the important characteristics of polymers if they are to be used as electrical insulators; in general, it should be minimal. Specifically, the conductivity of an insulator should stay low for long periods of time under electric field; this is important for many applications [1]. Experimentally, a strong dependence of electrode material on the conductivity suggests that the current is dominated by carriers injected from the electrodes [2,3]. A polymer composite is an inhomogeneous material and determination of its charge transport mechanisms is challenging [4]. Although some experimental studies report that the conductivity is decreased if inorganic particles are added [5-8] to polyethylene others report that it is increased $[9,10]$, all agree that the presence of particle-polymer interfaces in a composite affect the current flow. This may be due to the introduction or modification of the charge trapping sites. It has been shown [10] that while the current through the unfilled polymer decreases with time, all nanocomposites reveal an initial decrease, followed by a period in which the current increases with increasing time in a constant electric field and it is suggested that this may be related to increased charge mobility. However, other studies have suggested that the charge mobility is reduced in composites, due to charge trapping [5,7-9]. In recent years it has been noticed how particles draw moisture into the hydrophobic polymer matrix, degrading dielectric properties $[10,11]$. This absorbed water has been used as a probe to study polymer - particle interfaces [12]. In this paper, we report on an investigation into the conductivity behaviour of LDPE composites containing nanosilica, either untreated (hydroxyl surface chemistry) or treated using triethoxy(octyl)silane.

\section{Experimental}

Low density polyethylene LD100BW (ExxonMobil Chemicals) was used as the polymer matrix. Silica nanopowder (Sigma-Aldrich) with a quoted particle size range from 10 to $20 \mathrm{~nm}$ was used as the nanofiller. Materials were prepared as outlined previously [13]. Composites were formulated by solvent blending: one set using the nanosilica as-supplied, while the other set containing octyl-silane functionalised nanosilica. The filler loading level was confirmed using TGA.

\section{1 Electrical measurements}

DC-conductivity measurements were made using a constant voltage technique. In this, first the voltage was increased from $100 \mathrm{~V}$ to achieve a final $30 \mathrm{kV} / \mathrm{mm}$ constant electric field, in $100 \mathrm{~V}$ steps, and then the current was recorded over a period of time. Data were also acquired without any applied voltage, to differentiate the recorded signal from the background noise $( \pm 3 \mathrm{pA})$. When measuring very small currents, results can be easily affected by the noise from the environment and the measurement setup itself. Samples were $\sim 0.2 \mathrm{~mm}$ in thickness, onto which $30 \mathrm{~mm}$ diameter gold electrodes were sputter coated on each side; the diameter of solid spring loaded electrodes was $20 \mathrm{~mm}$. Fig. 1 shows the setup used in the DC-conductivity measurement. The oven contained a silica gel desiccant when a dry atmosphere was needed. The dielectric response was measured using a Solartron 1296 dielectric interface together with a Schlumberger SI 1260 impedance/phase 
gain analyser and a Solartron 12962A sample holder with a $30 \mathrm{~mm}$ diameter electrode. To collect the data, an ACvoltage of $1 \mathrm{~V}$ was applied and the frequency was swept from $0.1 \mathrm{~Hz}$ to $0.1 \mathrm{MHz}$ at 8 points per decade. Four samples of each material were tested to ensure reproducibility of the derived data. Prior to water immersion testing, the samples were dried in a vacuum oven at $80^{\circ} \mathrm{C}$ until no significant weight change occurred (one week). The samples were then immersed in distilled water and, at different time intervals, were removed, carefully dried with a tissue, weighed and subjected to dielectric spectroscopy testing. The sample mass was monitored using a precision balance; repeated measurements of a reference mass corresponded to a measurement uncertainty of about $\pm 0.05 \%$.

\section{Results and discussion}

Table 1 shows the composition of the studied materials and their water uptake after 30 days of water immersion. Fig. 2 shows average time dependent water uptake data for each material, from which it is evident that the unfilled polyethylene does not absorb water. While surface treatment of the nanosilica has a slight protective effect against water absorption, significant quantities of water are absorbed by both the systems containing nanosilica. The amount of absorbed water increases with increasing immersion time. From the data shown in Fig.2; it is evident that equilibrium water absorption is reached after 14 days of immersion.

Fig 3 shows representative conductivity/time curves for $8 \mathrm{wt} \%$ treated nanosilica composite $(\mathrm{PE} / \mathrm{C} 8 \mathrm{Si} / 8)$ under ambient and dry conditions; data obtained from an unfilled PE reference sample under dry conditions is shown, as a reference. From Fig. 3, the effect of the environmental conditions pertaining during the measurement duration for samples containing nanosilica is obvious. It takes approximately $1.5 \mathrm{~h}$ to notice the effect of humidity on conductivity. Fig. 4 shows the composites' conductivity response under different humidity levels: surface modification of the nanosilica cannot totally prevent the moisture penetrating into the composite. Indeed, based on the kinetics of water migration shown in Fig. 2, it would appear that only minute quantities of water have an effect on the bulk conductivity. Consequently, the conductivity increases

Table 1 - Studied materials and their moisture uptake.

\begin{tabular}{|l|c|c|c|}
\hline Sample Name & Filler & $\begin{array}{c}\text { Filler } \\
\text { load wt\% }\end{array}$ & $\begin{array}{c}\text { Mass gain after } \\
\text { 30 days in } \\
\text { water }(\%)\end{array}$ \\
\hline $\mathrm{PE}$ & - & - & $(0.3 \pm 0.5)$ \\
\hline $\mathrm{PE} / \mathrm{C} 8 \mathrm{Si} / 8$ & octyl-silica & 8 & $(73 \pm 11)$ \\
\hline $\mathrm{PE} / \mathrm{Si} / 7$ & silica & 7 & $(89 \pm 5)$ \\
\hline $\mathrm{PE} / \mathrm{C} 8 \mathrm{Si} / 4$ & octyl-silica & 4 & $(30 \pm 10)$ \\
\hline $\mathrm{PE} / \mathrm{Si} / 4$ & silica & 4 & $(47 \pm 8)$ \\
\hline
\end{tabular}

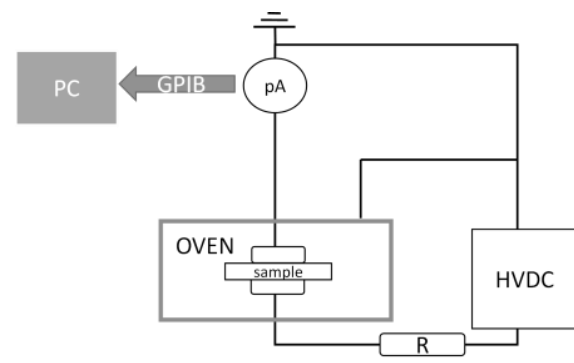

Fig 1. -DC conductivity measurement setup.

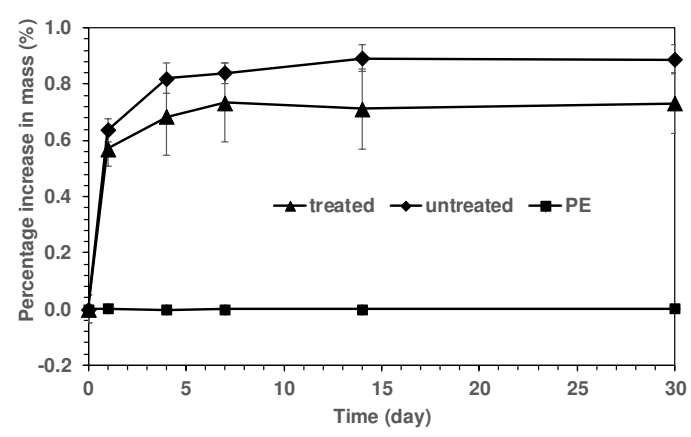

Fig. 2. - The averaged water uptake capability from 4 samples of: $\mathrm{PE} / \mathrm{C} 8 \mathrm{Si} / 8, \mathrm{PE} / \mathrm{Si} / 7$, and $\mathrm{PE}$ as reference. The error bars show standard deviation.

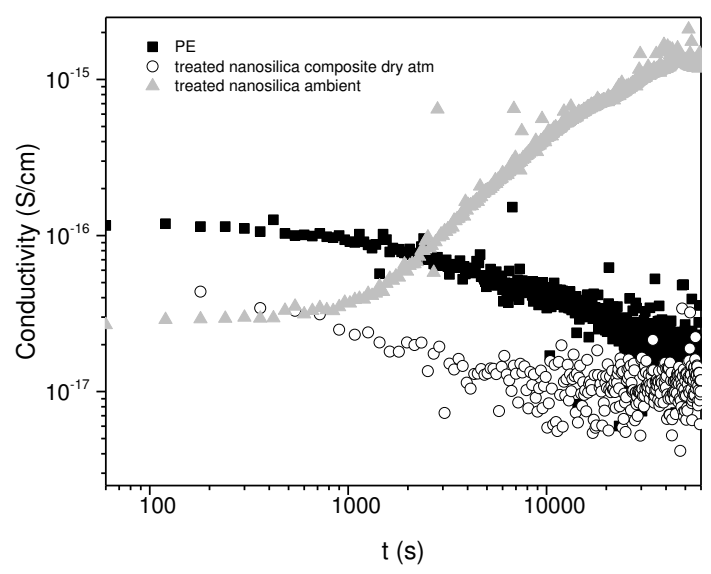

Fig. 3. - DC-conductivity of $\mathrm{PE}$ and $\mathrm{PE} / \mathrm{C} 8 \mathrm{Si} / 8$ composite in ambient and dry conditions.

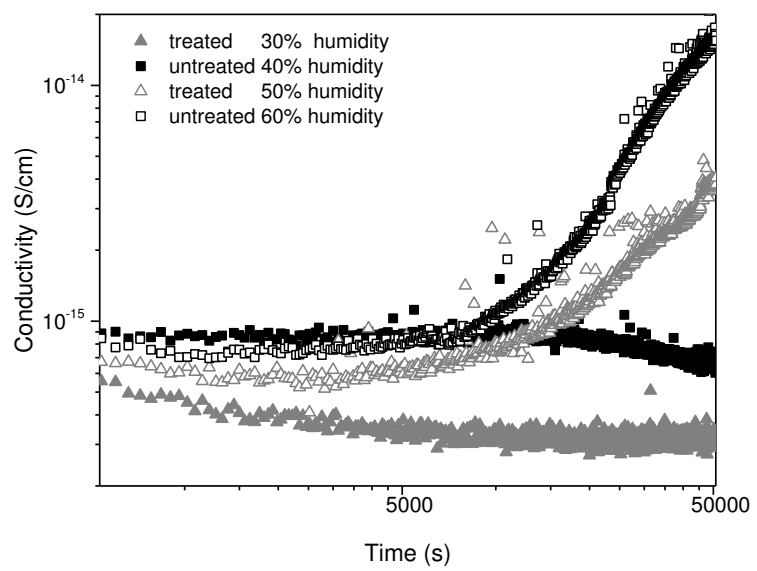

Fig. 4.- DC-conductivity of untreated silica composite $\mathrm{PE} / \mathrm{Si} / 7$ and treated nanosilica $\mathrm{PE} / \mathrm{C} 8 \mathrm{Si} / 8$ composite in different humidities. 

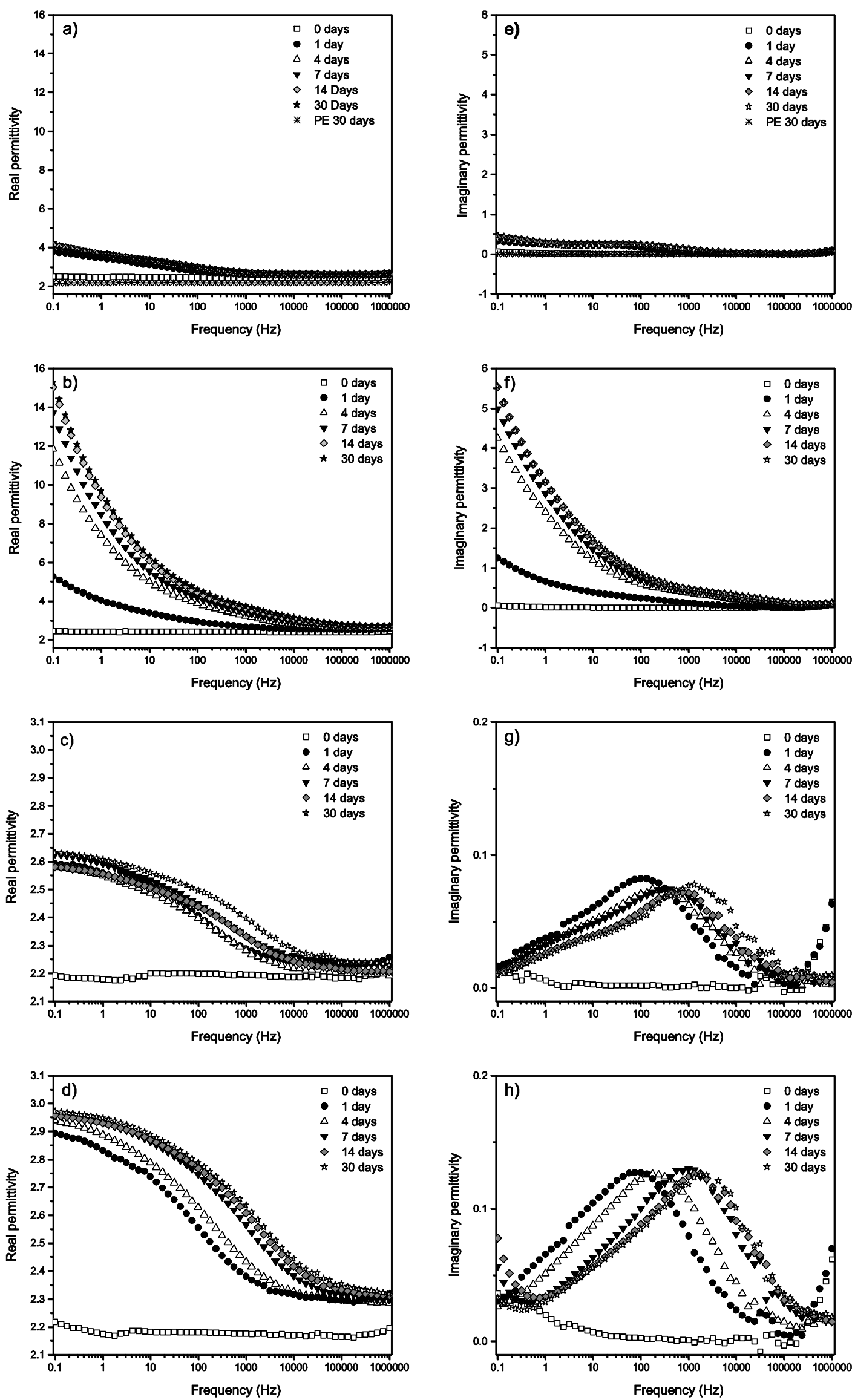

Fig. 5.-Real relative permittivity of nanocomposites containing a) $8 \mathrm{wt} \%$ of octylsilane treated nanosilica, b) $7 \mathrm{wt} \%$ of untreated nanosilica , c) 4 wt $\%$ of octylsilane treated nanosilica, d) $4 \mathrm{wt} \%$ of untreated nanosilica, and dielectric loss tangent of nanocomposites containing e) 8 wt $\%$ of octylsilane treated nanosilica f) $7 \mathrm{wt} \%$ of untreated nanosilica g) $4 \mathrm{wt} \%$ of octylsilane treated nanosilica h) $4 \mathrm{wt} \%$ untreated nanosilica upon water immersion at different time intervals (note the scaling difference between graphs from different filler loadings). 
with increasing humidity level. Several samples of each material were measured as there is quite a significant variation in the magnitude of conductivity from sampleto-samples, but the trend of increasing conductivity with increased humidity is always evident in silica-containing composites. This change in the slope in the conductivity has been previously linked to charge trapping at nanoparticle-polymer interfaces [7], or to enhanced charge mobility in the nanocomposites [10]. The work presented here strongly indicates that variations seen in the conductivity of the composite materials when compared to the polyethylene could be related to the fact that silica absorbs moisture from the surroundings.

Dielectric spectroscopy data obtained from samples of with increasing water content are shown in Fig. 5. The spectra for 30 day immersed pure system (PE) is also plotted for reference in Figs. 5a and 5e. The real or imaginary permittivity of the unfilled polyethylene do not change upon exposure to water remaining invariant up to 30 days of water immersion. Conversely, for composites containing both types of nanosilica, the dielectric response is changed compared to the unfilled system, with a huge increase in the real permittivity for $\mathrm{PE} / \mathrm{Si} / 7$, which corresponds to the higher filler loading of untreated silica sample used here. Prior to water immersion, the real permittivity of the nanocomposite sample does not vary significantly across the frequency range studied, (see Fig. 5 plot for 0 day). In the low frequency range, the slightly higher imaginary permittivity of the sample can be associated with the particle-polymer interfaces, where mobile charges can be trapped. With increased water absorption, the real permittivity increases and the peak in imaginary permittivity shifts to higher frequencies. But what is also seen, is that for sample $\mathrm{PE} / \mathrm{Si} / 7$ which contains the high loading of untreated nanosilica, low frequency losses increase dramatically. Indeed, the real part of the permittivity at $0.1 \mathrm{~Hz}$ for nanocomposite $\mathrm{PE} / \mathrm{Si} / 7$ reaches $\sim 15$, compared with $\sim 4$ for $\mathrm{PE} / \mathrm{C} 8 \mathrm{Si} / 8$ sample containing silica treated with octylsilane. This difference in permittivity between composites containing treated and untreated nanosilica is much more than if nanocomposites containing half of the amount of nanosilica; $\mathrm{PE} / \mathrm{Si} / 4$ and $\mathrm{PE} / \mathrm{C} 8 \mathrm{Si} / 4$ are compared to each other. (compare Fig. $5 \mathrm{c}$ ) and g) with $5 \mathrm{~d}$ ) and h), respectively). The increased low frequency losses originate from, firstly, that there will be increased amount of larger nanosilica clusters in the more highly filled samples [14]. In general, it is difficult to make meaningful conclusions on dielectric response on the structure if the structure itself cannot be thoroughly investigated. Secondly, the water distribution has changed: it seems that water shells at the filler interfaces are connected, resulting in a pseudo-DC conduction influencing the dielectric response.

These results give a broad perspective on be the dielectric frequency response of wet silica composites. Previous studies of comparable systems [12] have revealed relaxation peaks that shift to higher frequencies with prolonged wetting, which have been associated with differently bound layers of water on particle surfaces. However, no comparable increase in low frequency losses was reported with a similar material containing higher loading of same silica powder. The dielectric response shown here suggests that the particle phase has percolated when the filler loading is 8 or $7 \mathrm{wt} \%$, indicating that dielectric spectroscopy can be used also to probe the sample quality.

\section{Conclusions}

Low density polyethylene does not absorb moisture, but if either surface treated or untreated nanosilica is added, water absorption can have a dramatic effect on insulating properties. The dielectric response shows that surface treatment lowers the low frequency losses of wet material, but DC-conductivity will depend on the atmospheric humidity even if particle surfaces are treated - here, with octyl-silane. This study points out that when interpreting DC-conductivity results, the measurement conditions should be taken into consideration in the first instance, before making further conclusions about material characteristics.

\section{Acknowledgements}

The authors would like to thank the Engineering and Physical Science Research Council EPSRC for supporting this work through the Advances Polymer material for Energy Security - POLYMAT grant, EP/N002288/1. All the data supporting this study are openly available from the University of Southampton repository at doi:10.5258/SOTON/D0086.

\section{References}

[1] J. Lewiner, "Evolution of Experimental Techniques for the Study of the Electrical Properties of Insulating Materials" IEEE Trans. Dielectr. Electr. Insul., EI-21(3) 1986, pp.351-360.

[2] B. Sanden, and E. Ildstad, "DC conduction current and voltage endurance of polypropylene film" IEEE Conference on Electrical Insulation and Dielectric Phenomena, Annual Report, 1, 1998.

[3] J. Viertel, L. Petersson, A. Friberg, G. Dominguez, C. Tornkvist, "Electrode influence on DC conductivity measurements of low density polyethylene" IEEE Conference on Solid Dielectrics ICSD, 2013, pp.1048-1051

[4] R. Landauer, "Electrical conductivity in inhomogeneous media" Electrical transport and optical properties of inhomogeneous media, 40(1), 1978.

[5] R. Fleming, T. Pawlowski, A. Ammala, P. S. Casey and K. A. Lawrence, "Electrical conductivity and space charge in LDPE containing TiO2 nanoparticles", IEEE Trans. Dielectr. Electr. Insul., 12(4) 2005, pp. 745753.

[6] R. Fleming, A. Ammala, P. Casey and S. B. Lang, "Conductivity and space charge in LDPE containing nano-and micro-sized $\mathrm{ZnO}$ particles", IEEE Trans. Dielectr. Electr. Insul., 15(1), 2008, pp.18-125. 
[7] R.C. Smith, C. Liang, M. Landry, J.K. Nelson, and L.S. Schadler, "The mechanisms leading to the useful electrical properties of polymer nanodielectrics" IEEE Trans. Dielectr. Electr. Insul., 15(1), 2008, pp.187196.

[8] A. Bulinski, S. S. Bamji, M. Abou-Dakka, and Y. Chen, "Dielectric properties of polypropylene loaded with synthetic organoclay" IEEE Conference on Electrical Insulation and Dielectric Phenomena CEIDP, 2009, pp. 666-671.

[9] T. Tanaka, A. Bulinski, J. Castellon, M. Frechette, S. Gubanski, J. Kindersberger, ... and S. Pelissou, "Dielectric properties of XLPE/SiO2 nanocomposites based on CIGRE WG D1. 24 cooperative test results" IEEE Trans. Dielectr. Electr. Insul., 18(5) 2011, pp. 1482-1517.

[10] K. Y. Lau, A. S. Vaughan, G. Chen, I. L. Hosier, and A. F. Holt. "Absorption current behaviour of polyethylene/silica nanocomposites" Journal of Physics: Conference Series, vol. 472, no. 1, 2013, 012003.

[11] L. Hui, L. S. Schadler, and J. K. Nelson, "The influence of moisture on the electrical properties of crosslinked polyethylene/silica nanocomposites" IEEE Trans. Dielectr. Electr. Insul., 20(2), 2013, pp.641-653.

[12] K. Y. Lau, A. S. Vaughan, G. Chen, I.L. Hosier, and A.F. Holt, "On the dielectric response of silica-based polyethylene nanocomposites" J. Phys. D: Appl. Phys. 46, 2013, 095303.

[13] S. Virtanen, F. Saiz, A.S. Vaughan, N. Quirke, "Dielectric Breakdown Strength and Electrical Conductivity of Low Density Polyethylene Octylnanosilica Composite" IEEE Conference on Electrical Insulation and Dielectric Phenomena CEIDP, 2016, pp. $58-61$.

[14] S. Virtanen, H. Ranta, S. Ahonen, M. Karttunen, J. Pelto, K. Kannus and M. Pettersson, "The structure and dielectric properties of nano- $\mathrm{CaCO}_{3} /$ polypropylene composites" J. Appl. Pol. Sci., 131(1), 2014, 39504. 